\title{
Pion charge radius from charged pion electroproduction
}

\author{
Véronique Bernard, ${ }^{1, *}$ Norbert Kaiser, ${ }^{2, \dagger}$ and Ulf-G. Meißner ${ }^{3, \$}$ \\ ${ }^{1}$ Laboratoire de Physique Théorique, Université Louis Pasteur, F-67084 Strasbourg Cedex, France \\ ${ }^{2}$ Physik Department, Technische Universität München, D-85747 Garching, Germany \\ ${ }^{3}$ Institut für Kernphysik (Theorie), Forschungszentrum Jülich, D-52425 Jülich, Germany
}

(Received 29 March 2000; published 10 July 2000)

\begin{abstract}
We analyze a low-energy theorem of threshold pion electroproduction which allows one to determine the charge radius of the pion. We show that at the same order where the radius appears, pion loops induce a correction to the momentum dependence of the longitudinal dipole amplitude $L_{0+}^{(-)}$. This model-independent correction amounts to an increase of the pion charge radius squared from the electroproduction data by about $0.27 \mathrm{fm}^{2}$. It sheds light on the apparent discrepancy between the recent determination of the pion radius from electroproduction data and the one based on pion-electron scattering.
\end{abstract}

PACS number(s): 25.30.Rw, 14.40.Aq, 12.39.Fe

The charge (vector) radius of the pion is a fundamental quantity in hadron physics. It can essentially be determined in two ways. One method is pion scattering off electrons (or electron-positron annihilation into pion pairs), which leads to a pion root-mean-square (rms) radius of [1]

$$
\left\langle r_{\pi}^{2}\right\rangle_{V}^{1 / 2}=(0.663 \pm 0.006) \mathrm{fm}
$$

if one insists on the correct normalization of the pion charge (vector) form factor, $F_{\pi}^{V}(0)=1$ (in units of the elementary charge $e$ ). A more recent determination of the pion vector radius from low-momentum spacelike and timelike form factor data based on a very precise two-loop chiral perturbation theory representation [2] gives

$$
\left\langle r_{\pi}^{2}\right\rangle_{V}^{1 / 2}=(0.661 \pm 0.012) \mathrm{fm}
$$

consistent with the value given above. This number can be semiquantitatively understood in a naive vector meson dominance picture $\left\langle r_{\pi}^{2}\right\rangle_{V}^{1 / 2}=\sqrt{6} / M_{\rho} \simeq 0.63 \mathrm{fm}$. The second method is based on charged pion electroproduction $\gamma^{\star} p \rightarrow \pi^{+} n$. Here, $\gamma^{*}, p, n$, and $\pi^{+}$denote the virtual photon, proton, neutron, and positively charged pion, in order. The unpolarized cross section in parallel kinematics decomposes into a transversal and a longitudinal piece. ${ }^{1}$ While the former is sensitive to the the nucleon axial radius, the latter is quite sensitive to the pion form factor, i.e., to the pion radius for small momentum transfer. A recent measurement at the Mainz Microtron MAMI-II led to a pion radius of [4]

$$
\left\langle r_{\pi}^{2}\right\rangle_{V}^{1 / 2}=(0.74 \pm 0.03) \mathrm{fm}
$$

which is a sizably larger value than the one obtained from $\pi e$ scattering. It was hinted in Ref. [4] that their larger value for the pion radius might be due to the inevitable model

\footnotetext{
*Electronic address: bernard@1pt6.u-strasbg.fr

${ }^{\dagger}$ Electronic address: Norbert.Kaiser@Physik.TU-Muenchen.de

‡Electronic address: Ulf-G.Meissner@fz-juelich.de

${ }^{1}$ For a textbook discussion on this issue, we refer the reader to Ref. [3].
}

dependence based on the Born term approach to extract the pion radius. It was also stated that there might be an additional correction obtainable from chiral perturbation theory as is the case for the nucleon axial radius. Such a correction based on pion loop diagrams had been predicted in 1992 for the axial radius [5] and was verified by the MAMI experiment to a good precision. A list of references pertaining to previous determinations of the nucleon axial radius from (anti)neutrino-proton scattering and pion electroproduction can be found in Ref. [4]. Here, we show that there is indeed a similar kind of correction for the pion radius. This new term modifies the momentum dependence of the longitudinal $S$-wave amplitude $L_{0+}^{(-)}$and leads one to expect an even larger pion charge radius than the one given in Eq. (3). It is conceivable that higher order corrections yet to be calculated or contributions from higher multipoles will completely resolve the discrepancy between the pion radius determined from $\pi e$ scattering on one side and from charged pion electroproduction on the other.

Chiral perturbation theory allows one to make modelindependent statements based solely on the spontaneously and explicitly broken chiral symmetry of QCD. S-matrix elements and transition currents are systematically expanded in external momenta and quark (meson) mass insertions, collectively denoted by a small parameter $q$. Based on the underlying power counting, to a given order, one has to consider tree as well as pion loop graphs. Of particular interest are the so-called low-energy theorems, which give predictions to a certain order expressed entirely in terms of measurable quantities (a detailed discussion about this issue can be found in Ref. [6]). Our starting point is the low-energy theorem for the longitudinal dipole amplitude $L_{0+}^{(-)}$, accessible in charged pion electroproduction. ${ }^{2}$ It has been derived from baryon chiral perturbation theory to third order in the chiral expansion and reads [7]

\footnotetext{
${ }^{2}$ We use standard notation for the multipoles, the superscript refers to the isospin, the subscripts are $l \pm$, with $l$ the orbital angular momentum of the pion-nucleon system and the total angular momentum is $j=l \pm 1 / 2$. For a detailed discussion of the kinematics and multipole decomposition in pion electroproduction see Refs. $[3,7]$.
} 


$$
\begin{aligned}
& L_{0+}^{(-)}(\mu, \nu)= E_{0+}^{(-)}(\mu, \nu)+\frac{e g \pi N}{8 \pi m}\left(\mu^{2}-\nu\right)\left\{\frac{\kappa_{v}}{4}+\frac{m^{2}}{6}\left\langle r_{A}^{2}\right\rangle+\frac{\sqrt{(2+\mu)^{2}-\nu}}{2(1+\mu)^{3 / 2}\left(\nu-2 \mu^{2}-\mu^{3}\right)}\right. \\
&\left.+\left(\frac{1}{\nu-2 \mu^{2}}-\frac{1}{\nu}\right)\left(F_{\pi}^{V}\left(m^{2} \nu\right)-1\right)+\frac{m^{2}}{8 \pi^{2} F_{\pi}^{2}} \Xi_{4}\left(-\nu \mu^{-2}\right)\right\}+\mathcal{O}\left(q^{3}\right), \\
& E_{0+}^{(-)}(\mu, \nu)=\frac{e g_{\pi N}}{8 \pi m}\left\{1-\mu+C \mu^{2}+\nu\left(\frac{\kappa_{v}}{4}+\frac{1}{8}+\frac{m^{2}}{6}\left\langle r_{A}^{2}\right\rangle\right)+\frac{\mu^{2} m^{2}}{8 \pi^{2} F_{\pi}^{2}} \Xi_{3}\left(-\nu \mu^{-2}\right)\right\}+\mathcal{O}\left(q^{3}\right),
\end{aligned}
$$

with

$$
\begin{gathered}
\Xi_{3}(\rho)=\sqrt{1+\frac{4}{\rho}} \ln \left(\sqrt{1+\frac{\rho}{4}}+\frac{\sqrt{\rho}}{2}\right)+2 \int_{0}^{1} \sqrt{(1-x)[1+x(1+\rho)]} \arctan \frac{x}{\sqrt{(1-x)[1+x(1+\rho)]}}, \\
\Xi_{4}(\rho)=\int_{0}^{1} d x \frac{x(1-2 x)}{\sqrt{(1-x)[1+x(1+\rho)]}} \arctan \frac{x}{\sqrt{(1-x)[1+x(1+\rho)]}},
\end{gathered}
$$

in terms of the dimensionless quantities $\mu=M_{\pi} / m$ and $\nu$ $=k^{2} / m^{2}$, with $M_{\pi}(m)$ the charged pion (nucleon) mass and $k^{2} \leqslant 0$ the photon virtuality. Furthermore, $\kappa_{v}=\kappa_{p}-\kappa_{n}$ $=3.71$ is the isovector anomalous moment of the nucleon, $F_{\pi}=92.4 \mathrm{MeV}$ the weak pion decay constant, $g_{\pi N}=13.4$ the pion-nucleon coupling constant, and $C \simeq 0.4$ depends on some low-energy constants. Its precise form is not of interest here but can be found in Ref. [7]. We remark that these expressions have been obtained in a relativistic version of baryon chiral perturbation theory and that some of the kinematical prefactors have not been expanded. However, since we are only after a leading order pion loop effect, the result will not be different from a heavy baryon analysis [8,9]. For calculating higher order corrections consistently, one either has to use the heavy baryon formalism or the recently proposed infrared regularization [10]. Note also that the dependence of $L_{0+}^{(-)}$on the axial radius is very weak because the terms $\sim \nu\left\langle r_{A}^{2}\right\rangle$ cancel (that is why the axial radius discrepancy discussed before resides in the electric dipole amplitude [5]). As announced, the pion vector form factor $F_{\pi}^{V}\left(k^{2}\right)$ appears in the expression for the longitudinal multipole. The pion form factor has the following low-energy expansion:

$$
F_{\pi}^{V}\left(k^{2}\right)=1+\frac{1}{6}\left\langle r_{\pi}^{2}\right\rangle_{V} k^{2}+\mathcal{O}\left(k^{4}\right)
$$

Consequently, to separate the pion radius, one should consider the slope of the longitudinal multipole. For doing that, one has to expand the functions $\Xi_{3,4}\left(-\nu \mu^{-2}\right)$ in powers of $k^{2}=\nu m^{2}$ and pick up all terms proportional to $\nu$. This gives

$$
\begin{aligned}
\left.\frac{\partial L_{0+}^{(-)}}{\partial k^{2}}\right|_{k^{2}=0}= & \frac{e g_{\pi N}}{32 \pi m}\left\{\frac{1}{M_{\pi}^{2}}-\frac{1}{m M_{\pi}}+\frac{13}{8 m^{2}}+\frac{1}{3}\left\langle r_{\pi}^{2}\right\rangle_{V}\right. \\
& \left.+\frac{1}{32 F_{\pi}^{2}}\left(\frac{16}{\pi^{2}}-1\right)+\mathcal{O}\left(M_{\pi}\right)\right\} .
\end{aligned}
$$

The first four terms are standard [11], they comprise the conventional dependence on the pion vector radius, recoil effects and the dominant chiral limit behavior of the slope of the longitudinal multipole. The strong $1 / M_{\pi}^{2}$ chiral singularity stems from the $k^{2}$ derivative of the pion-pole term which appears already at leading order in the chiral expansion.

The last term in Eq. (9) originates from the so-called triangle and tadpole (with three pions coupling to the nucleon at one point) diagrams which are known to play a prominent role in pion photoproduction and electroproduction (see Fig. $1)$. The formal reason for the appearance of this modelindependent contribution at order $k^{2}$ is that one cannot interchange the order of taking the derivative at $k^{2}=0$ and the chiral limit $M_{\pi} \rightarrow 0$. Consequently, all determinations of the pion radius from electroproduction (based on tree-level amplitudes including nucleon and pion form factors) have "'measured"' the modified radius

$$
\left\langle\widetilde{r}_{\pi}^{2}\right\rangle_{V}=\left\langle r_{\pi}^{2}\right\rangle_{V}+\frac{3}{32 F_{\pi}^{2}}\left(\frac{16}{\pi^{2}}-1\right)
$$

The term on the right-hand side of Eq. (10) amounts to 0.266 $\mathrm{fm}^{2}$, a bit more than half of the squared pion rms radius $\left\langle r_{\pi}^{2}\right\rangle_{V} \simeq 0.44 \mathrm{fm}^{2}$. Therefore, from the longitudinal multipole alone, one expects to find a larger pion radius if one analyses pion electroproduction based on Born terms
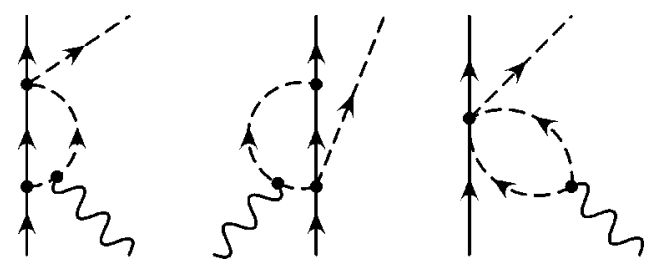

FIG. 1. Relevant one-loop diagrams. The solid, dashed, and wiggly lines denote nucleons, pions, and photons, in order. 


$$
\left\langle\widetilde{r}_{\pi}^{2}\right\rangle_{V}=(0.44+0.26) \mathrm{fm}^{2}=(0.83 \mathrm{fm})^{2},
$$

which is even larger than the result of the Mainz analysis, see Eq. (3). We point out, however, that the contribution of the pion radius to the derivative of the longitudinal multipole is a factor of 10 smaller than the one from the first three terms in the curly brackets in Eq. (9). Therefore, a fourth order analysis is certainly needed to further quantify the "pion radius discrepancy." Furthermore, the pion form factor contribution to the longitudinal cross section is also present in higher multipoles. In fact, it is known that the convergence of the multipole series for the pion pole term is slow. One should therefore also investigate such effects for these higher multipoles or directly compare the predictions of complete one-loop calculation with the data of the longitudinal electroproduction cross section. For the purpose of demonstrating the significance of chiral loop effects the $k^{2}$ slopes [considered here in Eq. (9) and in Ref. [5] for $E_{0+}^{(-)}$] are, however, best suited. What we have shown here is that as in the case of the nucleon axial mean square radius, the pion loops, which are a unique consequence of the chiral symmetry of QCD, modify the naive Born term analysis and should be taken into account.
[1] S. Amendolia et al., NA7 Collaboration, Nucl. Phys. B277, 168 (1986).

[2] J. Bijnens, G. Colangelo, and P. Talavera, J. High Energy Phys. 05, 014 (1998).

[3] E. Amaldi, S. Fubini, and G. Furlan, Pion Electroproduction (Springer-Verlag, Berlin, 1979).

[4] A. Liesenfeld et al., Phys. Lett. B 468, 20 (1999).

[5] V. Bernard, N. Kaiser, and Ulf-G. Meißner, Phys. Rev. Lett. 69, 1877 (1992).

[6] G. Ecker and Ulf-G. Meißner, Comments Nucl. Part. Phys. 21,
347 (1995).

[7] V. Bernard, N. Kaiser, T.-S.H. Lee, and Ulf-G. Meißner, Phys. Rep. 246, 315 (1994).

[8] E. Jenkins and A.V. Manohar, Phys. Lett. B 255, 558 (1991).

[9] V. Bernard, N. Kaiser, J. Kambor, and Ulf-G. Meißner, Nucl. Phys. B388, 315 (1992).

[10] T. Becher and H. Leutwyler, Eur. Phys. J. C 9, 643 (1999).

[11] S. Scherer and J.H. Koch, Nucl. Phys. A534, 461 (1991), and references therein. 